\title{
Asthma Diagnosis in Spain: Survey of Opinions, Attitudes and Knowledge among Primary Care Physicians
}

Jesus Molina Paris ${ }^{1 *}$, Vicente Plaza ${ }^{2}$, Miguel Angel Lobo Alvarez ${ }^{3}$, Xavier Muñoz $^{4}$, Manuel Pimentel Leal ${ }^{5}$ and Eduard Tarragona ${ }^{6}$

${ }^{1}$ Family and Community Medicine, Centro de Salud Francia, Fuenlabrada, Madrid, Spain

${ }^{2}$ Department of Respiratory Medicine, Hospital de la Santa Creu i Sant Pau, Institut d'Investigacio Biomedica Sant Pau (IIB Sant Pau), Department of Medicine, Universitat Autònoma de Barcelona, Barcelona, Spain

${ }^{3}$ Family Medicine, GRAP, EAP Gandhi, Madrid, Spain

${ }^{4}$ Pneumology Service, Hospital Universitari Vall d'Hebron, Barcelona, Spain

${ }^{5}$ Family and Community Medicine, EAP Torito, IMSALU, Madrid, Spain

${ }^{6}$ Department of Medical, Chiesi, Barcelona, Spain

\section{Abstract}

Background: Asthma is frequently misdiagnosed. Peak expiratory flow (PEF) measurement is easy and very useful for asthma diagnosis in primary care.

Objectives: The DIANA project aimed to assess the impact of a course on PEF measurement among primary care physicians in Spain.

Methods: A 7-item questionnaire was sent to selected primary care physicians (Phase A). Respondents were then invited to take a web-based course on PEF in asthma diagnosis. Finally, the questionnaire was again sent to all physicians (Phase B). Questionnaires also asked for demographical data such as age, sex, speciality, route of access to speciality, and geographical area.

A comparative statistical analysis was performed on the results of both questionnaires. An extended analysis was performed later. Answers were analysed by percentages and using McNemar's test. The planned duration of the project was one year.

Results: No statistically significant differences in percentages between the two phases were found for Items 1 (related to asthma diagnosis) and 4 (related to availability and use of PEF meter). However, for Item 2 (related to diagnosis of occupational asthma) differences were significant $(p<0.05)$, with an odds ratio $(O R)$ of 1.50 for those participating in Phase B. Differences were also significant $(p<0.05)$ for those who took the course, with an OR of 1.37. For Item 3 (related to the usefulness of measuring daily variability of PEF), there were also significant differences $(p<0.05)$ between the two phases, with an OR of 1.39. There were also significant differences $(p<0.05)$ for those who took the course, with an OR of 1.53 .

Conclusions: The first four results of the survey showed that educational intervention may improve knowledge of the usefulness of PEF in asthma diagnosis among primary care physicians. However, the high percentages of correct answers among those who did not take the course merit further investigation.

Keywords: Asthma; Diagnosis; Primary care; Peak expiratory flow rate; Medical education; Survey

\section{Introduction}

Asthma is one of the most frequent chronic respiratory diseases in primary care practice [1]. Asthma prevalence in Spain is increasing and, in children, is similar to that found in other European countries [2]. In a representative sample of the Spanish adult population, asthma prevalence was $4.8 \%$ (95\% CI 4.1-5.7) but reached $8.8 \%$ (95\% CI 6.911.2) in women over 65 years [3]. The majority of asthma patients are treated by primary care physicians [4].

According to asthma guidelines such as GINA (Global Initiative for Asthma) and GEMA (Guía Española para el Manejo del Asma, or Spanish Guide for Asthma Management), its diagnosis should be based on the history of variable respiratory symptoms (such as wheezing, shortness of breath, chest tightness and cough) and confirmed variable expiratory airflow limitation [2,5]. Excessive daily variability of lung function is essential for asthma diagnosis [5] and is assessed with peak expiratory flow (PEF) measurement $[2,5]$. This method is simple and convenient and is considered a useful diagnostic aid in primary care settings [1].

The DIANA (DIagnóstico de Asma eN Atención primaria, or Asthma Diagnosis in Primary Care) project sought to improve asthma patients' healthcare quality in primary care by means of a PEF training course and to assess its impact by means of a questionnaire. DIANA results will help to identify and describe areas of potential improvement and to offer recommendations for developing protocols that, together with clinical experience, facilitate healthcare practices in asthma in primary care settings.

\section{Methods}

\section{Study design}

The survey was developed as a 7-item questionnaire by two pneumologists. Items 1-4 were related to asthma diagnosis and the use of PEF meters, whereas items 5-7 were about the diagnosis of the last three asthma patients of the respondent. Questionnaires also asked for demographical data such as age, sex, speciality, route of access to

*Corresponding author: Jesus Molina Paris, Centro de Salud Francia, Calle de Francia, 38, 28943 Fuenlabrada, Madrid, Spain, Tel: +34916084215; E-mail jmolinaparis@gmail.com

Received September 15, 2015; Accepted December 29, 2015; Published December 31, 2015

Citation: Molina Paris J, Plaza V, Lobo Alvarez MA, Muñoz X, Pimentel Leal M, et al. (2015) Asthma Diagnosis in Spain: Survey of Opinions, Attitudes and Knowledge among Primary Care Physicians. J Pulm Respir Med 5: 308. doi:10.4172/2161 105X.1000308

Copyright: $\odot 2015$ Molina Paris J, et al. This is an open-access article distributed under the terms of the Creative Commons Attribution License, which permits unrestricted use, distribution, and reproduction in any medium, provided the original author and source are credited. 
speciality, and geographical area. The questionnaire was sent by mail to selected primary care doctors (Phase A). Answers were anonymous and voluntary.

Next, doctors who completed the survey were invited to take a 20-hour web-based course on the use of PEF in asthma diagnosis. The course was developed by pneumologists and primary care physicians. It included theoretical fundamentals, instructions for using the device, interpretation of PEF in asthma diagnosis and control, interpretation of PEF in special settings, and device maintenance. Additionally, it was accredited by the Spanish National Health System's Commission on Continuing Education.

After a period of nine months to allow this new knowledge to be implemented, the same questionnaire was again sent out to all selected doctors (Phase B).

\section{Selection of study subjects}

Participants had to be family doctors, general practitioners, paediatricians or other specialists practising in public or private primary care centres, who were used to looking after asthma patients, and who voluntarily consented to collaborate in the DIANA project.

The intended sample size was 1,000 primary care physicians to achieve a high degree of accuracy nationwide (with a maximum error of $\pm 5.6 \%$ for a $95 \%$ confidence interval and assuming the most unfavourable conditions of distribution of answers to dichotomous survey variables, $\mathrm{p}=\mathrm{q}=0.5$ ).

\section{Measurements}

Questionnaires were administered in paper format and designed to be read using an optical mark reader. The procedure was validated before closing the database for analysis. Once all the completed surveys from both phases had been received, they were processed by personnel specifically trained in electronic records management.

\section{Statistical analyses}

A comparative statistical analysis was performed on the results of both questionnaires. In an extended analysis, three tables were created for each item: the first with the percentage distribution of answers in each phase and the second and third with the number of right and wrong answers in each phase (the second table was created with the data from all participants and the third table with the data from those who took the training course). These last two tables allowed data to be contrasted using McNemar's paired data test (related samples). The chi-square test was not considered appropriate for the first table of each item because of the nature of the data (scores before and after training intervention). Additionally, a figure representing information on percentage analysis was calculated for each item. The figures also showed the magnitude and statistical significance level of the changes associated with taking the training course.

\section{Results}

This paper deals with results of the first four items of the questionnaires before and after the training course.

The study was carried out between June 2013 and January 2014. From the 1,000 doctors selected, 766 completed the first questionnaire, 633 completed both questionnaires, and 438 also took the PEF training course. Three questionnaires ( 2 from a nursing professional and 1 duplicated) were discarded. Furthermore, 2 questionnaires in Phase B were answered using a Phase A questionnaire and were shifted to Phase B. Thus, 762 Phase A questionnaires and 634 Phase B questionnaires were analysed.

The mean age of the participants was 46.8 years (95\% CI 46.1-47.4). There were 375 men (49.7\%) and 380 women (50.3\%); 7 professionals did not answer the question. Men were older than women: 49.0 years (95\% CI 48.1-50.0) vs. 44.5 years (95\% CI 43.6-45.4). Men were significantly older than women $(\mathrm{p}<00.5)$, with a median age of 52 years vs. 44 years in women.

With regard to speciality, $86.2 \%$ declared Community and Family Medicine, $11.7 \%$ general practitioners, $0.4 \%$ Internal Medicine and $1.7 \%$ other specialties; 8 professionals did not answer the question. Route of access to medical specialization was the medical specialists in training (MIR) system in $67.6 \%$ of participants; 45 professionals did not report their route of access. As far as geographical area of clinical practice was concerned, $21.4 \%$ worked in the Northern area, $22.7 \%$ in the Central area, $19.3 \%$ in the Southern area, and 3.2\% in Canary Islands, Ceuta and Melilla; 14 participants did not answer this question.

Table 1 shows the answer options for each item as well as the percentage distribution of answers in Phases A and B. Item 1 asked about which feature should preferably be used for asthma diagnosis; almost $60 \%$ of participants answered correctly Pattern of symptoms and variable airway obstruction in both phases. Item 2 related to which feature should preferably be used to diagnose occupational asthma; more than $40 \%$ of participants in Phase A and more than $50 \%$ in Phase 2 answered correctly Clinical symptoms (Pattern of symptoms and variable airway obstruction assessed with peak expiratory flow measurement at work). Item 3 asked about the usefulness of measuring daily variability of PEF with a PEF meter in customary clinical practice; almost $50 \%$ of Phase A participants and more than 55\% of Phase B answered correctly Maximum. Finally, Item 4 was about availability and use of a PEF meter; roughly $5 \%$ in both phases answered correctly I have one and always use it.

In Phase $\mathrm{A}$, there were significant differences in answers to items 2 and 4 according to gender of respondents $(\mathrm{p}<0.05)$ (data not shown). Answers to items 1 and 3 were not significantly different by gender. Moreover, there were significant differences $(\mathrm{p}<0.05)$ in answers to items 1 and 2 according to age (data not shown). Younger respondents (median age: 45 years) answered better than older (median age $>50$ years). There were no differences in answers to items 3 and 4 by age. By speciality, there was no significant difference in answers to items 1 to 4 (data not shown). However, by route of access to speciality, there were significant differences $(\mathrm{p}<0.05)$ only in item 1 . Respondents who accessed their specialization through the MIR system answered better (data not shown).

When participation in Phases A and B was compared, participants in both phases were younger (median age 47 years) than those who only answered the Phase A questionnaire (median age 50 years) $(\mathrm{p}<0.05)$. There was no difference by gender. In contrast, $87.8 \%$ of participants in both phases were Community and Family Medicine specialists vs. $78.5 \%$ in Phase A $(\mathrm{p}<0.05)$. However, there were no significant differences by age, sex or speciality between those who took the PEF course and those who did not.

Changes in answers between Phases A and B were also analysed to establish the impact of PEF course (data not shown). There were some noteworthy findings. In item 3 (usefulness of PEF measurement), 38 respondents ( 34 of them having taken the course) changed their answer to Maximum. In item 4 (availability and use of PEF meter), the answer I have one and use it frequently increased $23 \%$ among those who took the course. 
Citation: Molina Paris J, Plaza V, Lobo Alvarez MA, Muñoz X, Pimentel Leal M, et al. (2015) Asthma Diagnosis in Spain: Survey of Opinions, Attitudes and Knowledge among Primary Care Physicians. J Pulm Respir Med 5: 308. doi:10.4172/2161-105X.1000308

Page 3 of 5

Item

Item 1. Asthma diagnosis is preferably based on..

Phase A

(\%)

Specific history (Clinical diagnosis)

Pattern of symptoms and variable airway obstruction*

Objective demonstration of non-specific bronchial hyperresponsiveness

Measurement of bronchial inflammation

Symptom reversibility after therapy

Item 2. Diagnosis of occupational asthma is preferably based on...

Specific history (Clinical diagnosis)

Pattern of symptoms and variable airway obstruction assessed with peak expiratory flow (PEF) measurement at work*

Objective demonstration of non-specific bronchial hyperresponsiveness and/or assessment of bronchial inflammation at work

Reversibility of occupational asthma symptoms after withdrawing the patient from his/her working environment

Patient's referral for testing of specific bronchial hyperresponsiveness to a given agent that could induce occupational asthma

Item 3. Usefulness of measuring daily variability of expiratory flows with peak expiratory flow (PEF) meter in customary clinical practice

None

Low

Intermediate or moderate

Maximum*

Item 4. Availability and use of PEF meter

I neither have nor use one

I have one but do not use it

I have one but use it only occasionally

I have one and use it frequently

I have one and always use it ${ }^{\star}$

*right answer

Table 1: Percentages of answers to items 1-4

\section{Extended analysis}

The extended analysis did not found statistically significant differences in percentages between the two phases for item 1, even when data were stratified for those who took the PEF course (Figure 1).

For item 2, statistically significant differences $(\mathrm{p}<0.05)$ were found between the right answers in Phase A and Phase B. Participation in Phase B resulted in an odds ratio of 1.50 (95\% CI 1.16-1.94). Among the physicians who took the PEF course, differences were also statistically significant $(\mathrm{p}<0.05)$ (Figure 2), with an odds ratio of 1.37 (95\% CI 1.001.86).

Statistically significant differences $(p<0.05)$ were found among the right answers to item 3 in Phases A and B. Participation in Phase B implied an OR of 1.39 (95\% CI 1.06-1.84). When analysing data from those who took the training course, statistically significant differences $(\mathrm{p}<0.05)$ between Phases A and B were also found (Figure 3$)$. The OR was 1.53 (95\% CI 1.10-2.15).

Finally, no statistically significant differences were identified in percentages between Phases A and B for item 4, even when stratifying the answers according to those who took the training course. In Phase $\mathrm{B}$, the percentage of respondents who did not have and did not use a peak flow meter diminished considerably, while the percentage of respondents who had the device and used it occasionally or frequently increased. However, these differences did not achieve statistical significance (Figure 4).

\section{Discussion}

\section{Main findings}

This paper presents the results of the first four items in Phases A and $\mathrm{B}$. We did not find statistically significant differences between

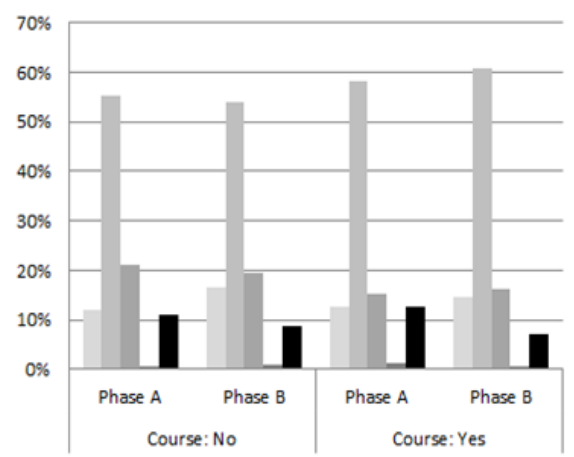

Specific history

Symptom pattern and variable aiflow obstruction (Right answer)

Objective demonstration of airway hyperresponsivenes

Me asurement of bronchia inflammation

Symptom reversibility afte therapy

Figure 1: Percentages of answers to Item 1, Asthma diagnosis is preferably based on...

Phases A and B in terms of knowledge of the main features for asthma diagnosis. This lack of difference may be due to the fact that the webbased course was focused on PEF, not on asthma.

With regard to diagnosis of occupational asthma, there were statistically significant differences: the percentage of right answers in Phase B was 1.5 times higher than in Phase A for all respondents irrespective of their taking the PEF course. Moreover, respondents who took the course had a percentage 1.37 times higher than those who did not. We hypothesize that this finding may be due to a recall effect when answering the questionnaires.

We found statistically significant differences between the two phases regarding the usefulness of PEF measurement in customary clinical practice. The score in Phase B was 1.39 times higher than in Phase A. Also, respondents who took the course had a score 1.53 times higher than those who did not. This finding supports the usefulness of 


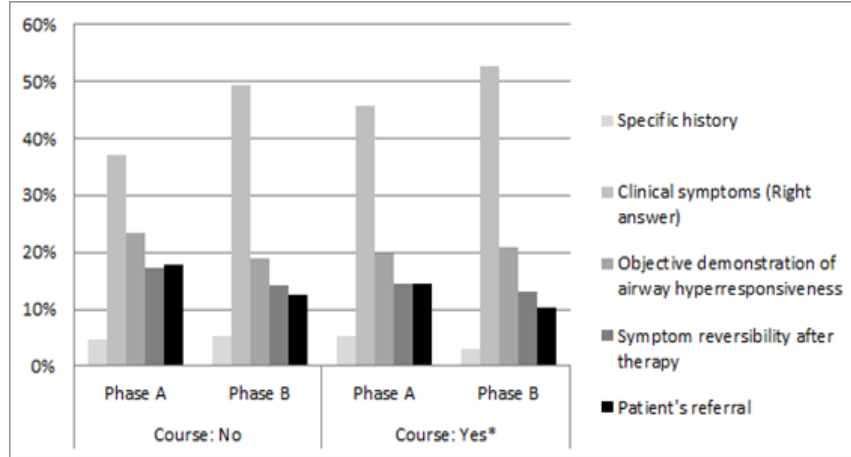

Figure 2: Percentages of answers to Item 2, Diagnosis of occupational asthma is preferably based on... ${ }^{*} p<0.05$.

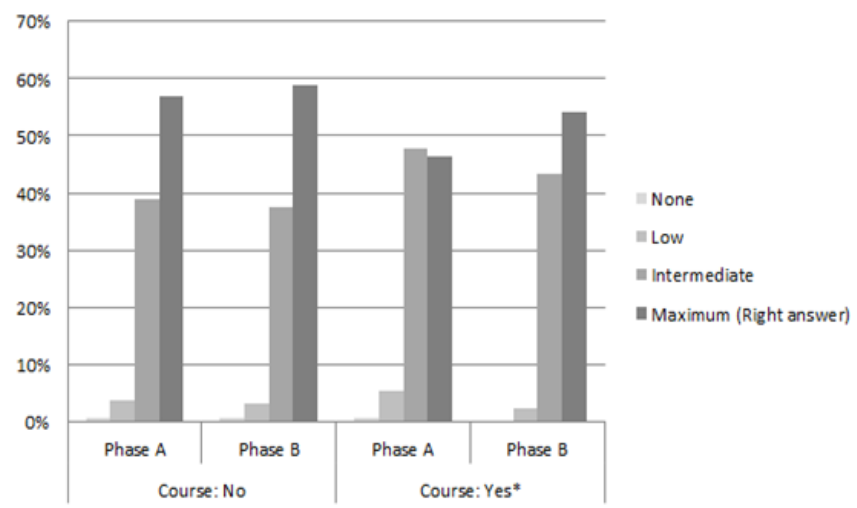

Figure 3: Percentages of answers to Item 3, Usefulness of measuring daily variability of expiratory flows with a peak flow meter in customary clinical practice. ${ }^{*} \mathrm{p}<0.05$.

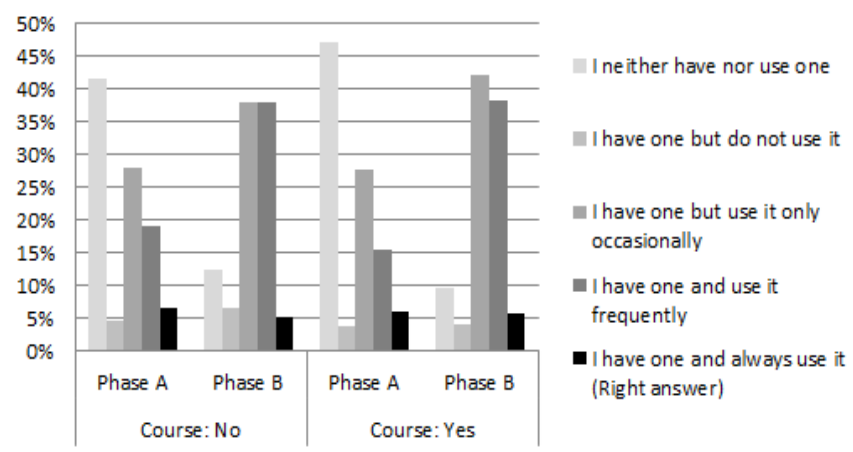

Figure 4: Percentages of answers to Item 4, Availability and use of peak expiratory flow meter.

\section{the PEF course.}

Conversely, there were no statistically significant differences between the two phases in the availability and use of PEF meter. However, the percentages of respondents who had a PEF meter and used it occasionally or frequently increased in Phase B, especially among those who took the course. This finding also supports the usefulness of the PEF course.

A surprising finding was that Phase $B$ results were also high among those who did not take the PEF course; for example, in items 3 and 4. We did not ask why the physicians surveyed took the course or refused to take it, but we hypothesize that several of those who refused the course already had a good or acceptable knowledge of PEF and asthma diagnosis. Age differences between those who took the course and those who did not may support this explanation, with older physicians being more experienced. Another possibility is that younger physicians are more disposed to update and increase their knowledge. Furthermore, it may be possible that the first survey motivated physicians to go over their knowledge of PEF and asthma, thus improving their results; for example, the increased availability and use of a PEF meter in Phase B. Conversely, it is possible that those who took the course were aware that they needed to enhance their knowledge. These unexpected results require further investigation.

\section{Strengths and limitations of this study}

The main strength of the DIANA project is that it is a nationwide study that included primary care physicians of different ages, specialities and geographical areas. Because of this, we regard its results as being applicable to all primary care professionals in Spain. By means of a simple questionnaire and an educational intervention, the DIANA project showed that it is possible to increase physicians' knowledge and awareness of PEF measurement and, thus, to improve asthma diagnosis in primary care.

Another strength is that we used a web-based course, not a printed or face-to-face course. According to a systematic review and later studies, web-based continuing medical education is effective in improving and maintaining physicians' performance [6,7]. In contrast, printed educational materials are among the least effective methods [6].

Moreover, our analysis of answers according to respondents' gender, age, speciality (family doctors, paediatricians, etc.), route of access to speciality, and geographical area could help to design more accurate educational interventions.

One potential limitation of the study is that participants were selected according to pre-established criteria and their inclusion was not randomized. However, we considered that the findings were applicable to primary care in Spain because of the large sample size, the statistical strength, and the distribution of physicians all over the country.

Another potential limitation, especially for items 3 and 4 , is that the answers to the questionnaires depended on the respondents' truthfulness and may not match actual clinical practice. However, future results of items 5 to 7 will help provide a more complete picture.

Interpretation of findings in relation to previously published work

In a Spanish survey of 1,066 physicians and nurses, 401 primary care physicians were included. Among the latter, $45 \%$ never or rarely applied asthma guidelines such as GINA, GEMA and others, and only $10-17 \%$ adhered closely to the GEMA guidelines. However, $48 \%$ were familiar with the GINA guidelines and 55\% with the GEMA guidelines [8]. We did not ask specifically for guideline knowledge, but $41.5 \%$ of respondents in Phase A and $41 \%$ in Phase B did not answer the item on asthma diagnosis correctly. Taken as a whole, these findings point to a lack of knowledge and application of asthma guidelines in Spain. Therefore, educational interventions developed from the results of surveys and projects like ours are needed.

As far as we know, there is only one other study of the use of PEF for asthma diagnosis in Spain. In a survey conducted among primary care physicians in the autonomous community of Galicia (Spain), 
Citation: Molina Paris J, Plaza V, Lobo Alvarez MA, Muñoz X, Pimentel Leal M, et al. (2015) Asthma Diagnosis in Spain: Survey of Opinions, Attitudes and Knowledge among Primary Care Physicians. J Pulm Respir Med 5: 308. doi:10.4172/2161-105X.1000308

$57 \%$ of respondents had a PEF meter $(28 \%$ had purchased the device for themselves) and $31 \%$ had used it within the last year. The more common indications were asthma monitoring and diagnosis $(45 \%$ and $31 \%$, respectively). Medical education needs were identified: $33 \%$ of respondents had never received specific PEF training and $38 \%$ had received it more than three years ago [9]. Although we did not specifically ask about the use of the PEF meter within the last year, $54.4 \%$ of respondents in Phase A and $89.5 \%$ in Phase B had access to a PEF meter. However, only $5.8 \%$ and $5.5 \%$, respectively, always used the device. Nevertheless, the PEF course was associated with an increased use of PEF.

\section{Implications for clinical practice}

Our findings have implications for asthma diagnosis in clinical practice. Asthma may be overdiagnosed or misdiagnosed. Furthermore, an incorrect diagnosis may explain the poor control of asthma in some patients [10]. In a Spanish study of patients receiving inhaled therapy in primary care, the majority of asthma patients were misdiagnosed according to current guidelines [11]. In two studies, up to $30 \%$ of patients with a physician diagnosis of asthma were actually misdiagnosed [12,13]. Moreover, chronic obstructive pulmonary disease (COPD) is often misdiagnosed as asthma in primary care [14] because differential diagnosis can be difficult [15]. Furthermore, differential diagnosis of asthma vs. COPD is essential for appropriate therapy [15]. However, most patients can be accurately diagnosed by primary care physicians following the current guidelines [15].

A better knowledge of guidelines such as GINA and GEMA, and of diagnostic tests such as PEF, would improve asthma diagnosis. Only approximately $50 \%$ of the DIANA respondents answered that measurement of daily variability by means of a PEF meter has maximum usefulness for asthma diagnosis. Furthermore, almost half of respondents did not have a PEF meter. However, PEF course increased availability and use of PEF meter.

Moreover, occupational asthma is underdiagnosed in primary care. Patients diagnosed with asthma $(n=368)$ in Spanish primary care centres completed a questionnaire that included their entire working history. An expert in occupational asthma assessed the answers and classified the patients as suffering from common asthma (60.8\%), occupational asthma (18.2\%) and work-exacerbated asthma (14.7\%). Therefore, $32.9 \%$ of patients had work-related asthma [16]. A statistically significant increase in the knowledge of occupational asthma diagnosis was seen in Phase B of the DIANA project, thus showing that medical education initiatives could improve the identification of this type of asthma.

Primary care physicians have an essential role in the early diagnosis of asthma, as well as in management and follow-up. Our project emphasizes the role of PEF in asthma and could contribute to improving asthma diagnosis and management in Spain.

\section{Conclusion}

The first results of the DIANA project showed that medical education has the potential to improve asthma diagnosis in primary care. However, the high percentages of correct answers among those who did not take the course merit further investigation.

\section{Acknowledgements}

Editorial assistance was provided by Content Ed Net, Madrid, Spain.

\section{Conflicts of Interest}

Jesús Molina París has received honoraria for speaking at sponsored meetings from AstraZeneca, Boehringer-Ingelheim, Chiesi, GlaxoSmithKline, Mundipharma, Novartis, Pfizer, Rovi, and Teva.

Vicente Plaza declares that in the last three years he has received honoraria for speaking at sponsored meetings from AstraZeneca, Boehringer-Ingelheim Chiesi, GlaxoSmithKline, Merck, Mundipharma, and Pfizer; and as a consultan for Mundipharma, Orion, and Teva. He has received support from BoehringerIngelheim and Chiesi for attending meetings. He also received funding/grant support for research projects from a variety of Government agencies and not-forprofit foundations, as well as Chiesi, Menarini, and Merck.

Eduard Tarragona is an employee of Chiesi.

\section{Financial Resources}

This study was supported by Chiesi.

\section{References}

1. Kaicker J, Dang W, D'Urzo A (2014) The challenge of objective confirmation of asthma diagnosis in primary care. NPJ Prim Care Respir Med 24: 14032.

2. http://www.gemasma.com/

3. Garin N, Olaya B, Perales J, Moneta MV, Miret M, et al. (2014) Multimorbidity patterns in a national representative sample of the Spanish adult population. PLoS One 9: e84794.

4. Levy ML, Fletcher M, Price DB, Hausen T, Halbert RJ, et al. (2006) Internationa Primary Care Respiratory Group (IPCRG) Guidelines: diagnosis of respiratory diseases in primary care. Prim Care Respir J 15: 20-34.

5. http://www.ginasthma.org.

6. Mostofian F, Ruban C, Simunovic N, Bhandari M (2015) Changing physician behavior: what works? Am J Manag Care 21: 75-84.

7. Lehr AR, McKinney ML, Gouin S, Blais J-G, Pusic MV, et al. (2013) Development and pretesting of an electronic learning module to train health care professionals on the use of the Pediatric Respiratory Assessment Measure to assess acute asthma severity. Can. Respir. J 20: 435-441.

8. Plaza V, Bolívar I, Giner J, Llauger MA, López-Viña A, et al. (2008) [Knowledge of and attitudes and adherence to the Spanish Guidelines for Asthma Management (GEMA) among Spanish health care professionals: the GEMA test Project]. Arch Bronconeumol 44: 245-251.

9. Romero de Ávila G, Gonzálvez Rey J (2015) [FEMGALICIA 2012 Study: Knowledge and management of peak espiratory flow meter in Galician Primary Care]. Cad Aten Primaria 21: 5-9.

10. Ringsberg KC, Bjärneman $P$, Larsson $R$, Wallström $E$, Löwhagen $O$ (2014) Diagnosis of asthma in primary health care: a pilot study. J Allergy (Cairo) 2014: 898965

11. Izquierdo JL, Martín A, de Lucas $P$, Rodríguez-González-Moro JM, Almonacid C, et al. (2010) Misdiagnosis of patients receiving inhaled therapies in primary care. Int J Chron Obstruct Pulmon Dis 5: 241-249.

12. Aaron SD, Vandemheen KL, Boulet LP, Mclvor RA, Fitzgerald JM, et al. (2008) Overdiagnosis of asthma in obese and nonobese adults. CMAJ 179: 11211131.

13. Luks VP, Vandemheen KL, Aaron SD (2010) Confirmation of asthma in an era of overdiagnosis. Eur Respir J 36: 255-260.

14. Tinkelman DG, Price DB, Nordyke RJ, Halbert RJ (2006) Misdiagnosis of COPD and asthma in primary care patients 40 years of age and over. $J$ Asthma 43: $75-80$

15. Miravitlles M, Andreu I, Romero Y, Sitjar S, Altés A, et al. (2012) Difficulties in differential diagnosis of COPD and asthma in primary care. $\mathrm{Br} \mathrm{J}$ Gen Pract 62: e68-75.

16. Vila-Rigat R, Panadès Valls R, Hernandez Huet E, Sivecas Maristany J Blanché Prat X, et al. (2015) Prevalence of Work-Related Asthma and its Impact in Primary Health Care. Arch Bronconeumol 51: 449-455. 